\title{
An Error Bound for Quadratures
}

\author{
By J. H. Hetherington
}

\begin{abstract}
A definition of a length scale is given for analytic functions. Quadrature error bounds based on this length scale can be used to compare different orders and types of quadrature rules.
\end{abstract}

A familiar theorem about convergence of Taylor series states that an analytic function has a bound on its derivatives so that, if the nearest singularity is at a radius greater than $r$ from the point $x$ in the complex plane, then there exists some $M$ such that, for all $n$,

$$
\left|f^{(n)}(x)\right|<M n ! r^{-n} \text {. }
$$

We need a lemma easily derived from the theorem which states: For all $M \geqq$ $|f(x)|$, and $M \neq 0$, there exists an $r$ greater than zero and less than the distance to the nearest singularity such that Eq. (1) holds for all $n$. The lemma is proved as follows: Define $r_{0}$ as a radius less than the distance to the nearest singularity, and define $M_{0}$ as an $M$ which satisfies (1) for $r=r_{0}$ according to the theorem. Then it is straightforward to show that, given an $M, M \geqq|f(x)|$ and $M>0$, an $r$ which satisfies the lemma is given by $r=\min \left(r_{0}, M r_{0} / M_{0}\right)$.

We now define two functionals of functions analytic in the neighborhood of a given interval. The first functional, $M$, is the maximum absolute value of the function on the interval under consideration. The second functional $r$ is the largest value $r$ which satisfies (1) for the given function for every $n$ and for every value $x$ in the interval when $M$ is as defined above in this paragraph. The lemma assures that such an $r$ exists. This value $r$ will be smaller than (or equal to) the smallest radius of convergence of the function as measured from points along the interval. The functional $r$ is valuable because it can be used as a scale of length for the "variability" of the function. A search for other useful definitions of scale might be valuable, but no other definition will be considered here.

If a quadrature rule over this interval has a degree of precision $(n-1)$, then the error for that rule can be written

$$
E_{n}=H^{n+1} G_{n} f^{(n)}(\xi),
$$

where $E_{n}$ is the error, $H$ is the interval length, $G_{n}$ a numerical coefficient and $f^{(n)}(\xi)$ is the $n$th derivative of the integrand evaluated at some point in the interval [1]. Eliminating $\left|f^{(n)}(\xi)\right|$ between (2) and (1), we obtain

$$
\left|E_{n}\right| / H M<\nu^{n} G_{n} n !(r \nu / H)^{-n} .
$$

Here $\nu^{n}$ has been introduced in the numerator and denominator. If $\nu$ is taken as the

Received July 16, 1971, revised November 3, 1971.

AMS 1969 subject classifications. Primary 6555, 6580.

Key words and phrases. Quadrature, error bound.

Copyright $\Subset$ 1972, American Mathematical Society 
number of panels (i.e., $\nu=$ number of points for open quadratures and $\nu+1=$ number of points for closed quadratures), then the quantity in parentheses in Eq. (3) is just a nondimensional average density of points which we will call the sketchability. Since $H M$ is a bound on the integral, the quantity on the left of (3) is seen to be the error expressed as a fraction of that bound. We will call the quantity $\left|E_{n}\right| / H M$ the fractional error. The form of Eq. (3) means that each quadrature rule will yield a straight line on a log-log plot of fractional error versus sketchability. One can thus compare the fractional error bounds for a variety of quadrature schemes as a function of the sketchability.

It is easily shown that the same bound applies whether the quadrature is applied to the whole interval or whether the interval is broken into uniform subintervals and the same quadrature is applied to each subinterval.

We have made comparisons for a few kinds of quadrature and find, because of the character of the numbers $G_{n}$, that among quadrature schemes of given types, say the odd closed Newton-Cotes schemes, different orders give the best error bound for a given sketchability in different sketchability regions. That is, for any given fractional error, one order of the quadrature is found to give the error bound desired with the

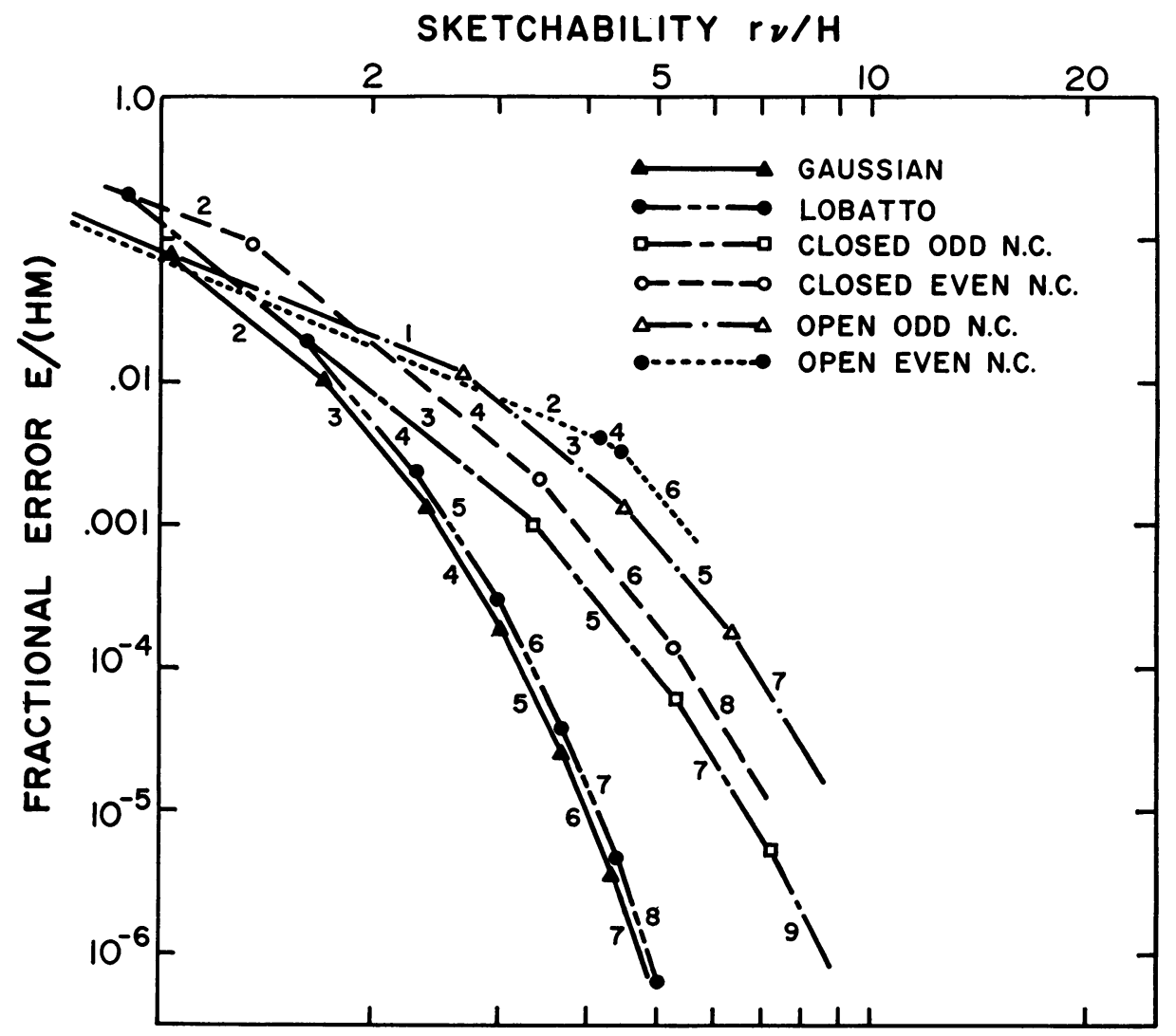

FIGURE 1. log-log plot of the "fractional error" bound $\left|E_{n}\right| /(H M)$ as a function of the "sketchability" $r \nu / H$ for several quadrature schemes. 
lowest sketchability, and the order which is best depends on the desired accuracy.

Fig. 1 shows the relationships of various low-order quadrature schemes. The straight line segments in Fig. 1 each correspond to Eq. (3), evaluated for some particular quadrature scheme. The intersection of the straight line with the line $r \nu / H=1$ is given by the quantity $\nu^{n} G_{n} n$ !, while the slope of the line is determined by $-n$. To prevent confusion, only that segment of each line is shown which gives a stronger bound than any of the other members of its family.

When comparing two quadrature schemes the scheme with the lowest sketchability for a given error is best and therefore lines to the left in Fig. 1 are stronger bounds. Numerical integration of any function analytic in the neighborhood of a given interval will yield a point on Fig. 1. The point will always lie to the lower left of the straight line which corresponds to the quadrature scheme used.

Examination of Fig. 1 shows that some order Gaussian quadrature scheme for most error ranges gives the strongest bound.

These bounds allow one to discriminate between the use of a single higher-order scheme or several lower-order schemes on a given interval. They show that, in general, lower-order schemes are better for obtaining low accuracy with fewer points while higher accuracy requires higher-order quadrature schemes [2]. This qualitative behavior will be preserved in any stronger error bounds, at least for the closed odd Newton-Cotes quadratures, because of the following considerations.

First, the integral $\int_{-1}^{1}\left(64+x^{2}\right)^{-1} d x$, calculated by a single Simpson's rule, has sketchability 8 and fractional error $3.2 \times 10^{-5}$. This establishes a lower limit for Simpson's rule bounds well above the nine-point closed Newton-Cotes bound of $2.1 \times 10^{-6}$ for this sketchability. On the other hand, the integral $\int_{-1}^{1}\left(.25+x^{2}\right)^{-1} d x$, calculated by a single nine-point closed Newton-Cotes quadrature, has sketchability 2 and fractional error .0123 which is above the Simpson's rule bound of .0083 for this sketchability. Thus, any stronger bounds for Simpson's rule and nine-point closed Newton-Cotes quadrature must intersect.

Since we have not found similar examples among the Gaussian quadratures, we cannot be assured that such intersections occur for the strongest bounds for that family of quadratures. A search for stronger bounds should be interesting.

A nonrigorous estimate of $r$ based on differences of the function has been found useful: $r$ can be estimated by applying Eq. (1) to several derivatives estimated from differences. Each such application of Eq. (1) gives a radius $r$, and the estimate of $r$ is the smallest radius so calculated. It has been found possible to construct an automatic adaptive quadrature scheme, based on this error bound, using this estimate of $r$. The method is to estimate $M$ as the largest value of $|f|$ on points collected on the interval. If $M$ and the desired error are known, it is possible to decide from Fig. 1 which order quadrature scheme is best among the family which is used (closed odd Newton-Cotes in the program which has been written). The interval can be subdivided if the estimate of $r$ based on differences does not indicate convergence. Thus, both the interval size and order of quadrature are adjusted.

It is straightforward to derive a similar error bound for interpolation schemes. The error bounds for various order Lagrangian interpolations on uniformly spaced points have been calculated. Similar behavior is found, i.e., lower orders give stronger bounds, when low accuracy is required; higher orders are better for high accuracy. 
Note added in proof. The error line for two-point open Newton-Cotes quadratures should lie a factor 3 above the line shown in Fig. 1 because of a misprint in Eq. 25.4.21 of reference 1 .

\section{Physics Department}

Michigan State University

East Lansing, Michigan 48823

1. M. Abramowitz \& I. A. STEgun (Editors), Handbook of Mathematical Functions with Formulas, Graphs, and Mathematical Tables, Nat. Bur. Standards Appl. Math. Series, 55, Superintendent of Documents, U.S. Government Printing Office, Washington, D.C., 1964. MR 29 \#4914.

2. Similar behavior has been found experimentally by K. E. Hillstrom, Comm. ACM, v. 13,1970, p. 362 . 